\title{
Auditoría social en las organizaciones del sector cooperativo: precedente de notable escenario de gobernabilidad
}

\author{
Gustavo Adolfo Rubio-Rodríguez ${ }^{1}$ - y Fernando de Almeida $\operatorname{Santos}^{2}{ }^{\complement}$
}

Recibido: 22 de enero de 2020 / Aceptado: 10 de diciembre de 2020 / Publicado: 24 de marzo de 2021

Resumen. Las organizaciones cooperativas forman un sector cada vez más visible y relevante, cuyo fin afianza la política de ofrecer respuesta a necesidades concretas de bienestar de las personas, colectividades y de la sociedad en general. El presente trabajo tiene por objeto resaltar la importancia que representa la auditoría social en las organizaciones del sector cooperativo, como respuesta derivada de diligentes prácticas de gobernabilidad y prácticas de buen gobierno; para ello, se tomaron 7 cooperativas especializadas de ahorro y crédito definidas por la legislación colombiana como una asociación autónoma de personas que se han unido voluntariamente para hacer frente a sus insuficiencias y aspiraciones económicas, sociales y culturales. Los hallazgos enseñan que la auditoría, cuando es administrada cabalmente, forja el cumplimiento de los objetivos estratégicos en materia social, esto es, utilizar los recursos de los fondos de destino específico en actividades propias de los mismos, tales como educación formal, salud, auxilios funerarios y otras actividades para el bienestar del asociado; actividades debidamente aprobadas por el órgano competente, reveladas en sus informes de gestión e información financiera. Como conclusión, la gobernabilidad de las cooperativas estudiadas sustenta su andamiaje en el acierto de la elección de los miembros de los comités de auditoría, quienes ejercen un efectivo control sobre los recursos con asignación específica.

Palabras clave: Economía social; Modelo de gobernanza; Organización cooperativa; Responsabilidad social; Sector solidario.

Claves Econlit: B55; M14; Q13.

\section{[en] Social auditing in the organizations of the cooperative sector: precedent of remarkable governance scenario}

\begin{abstract}
Cooperative organizations form an increasingly visible and relevant sector, whose purpose strengthens the policy of providing a response to specific welfare needs of individuals, communities and society in general. The purpose of this paper is to highlight the importance of social auditing in cooperative sector organizations, as a response derived from diligent governance practices; to this end, seven specialized saving and credit cooperatives defined by Colombian legislation as an autonomous association of persons who have voluntarily joined together to address their economic, social and cultural insufficiencies and aspirations were taken. The findings show that auditing when fully administered, forges the fulfilment of strategic social objectives, i.e. to use the resources of the specific earmarked funds in their own activities, such as formal education, health, funeral aid and other partner welfare activities; duly approved by the competent body, disclosed in its management reports and financial information. As a conclusion, the governance of the cooperatives studied underpins their scaffolding on the success of the choice of the members of the audit committees, who exercise effective control over the resources with specific allocation.
\end{abstract}

Keywords: Social economy; Governance model; Cooperative organization; Social responsibility; Solidary sector.

Sumario. 1. Introducción. 2. Acepción e importancia de la auditoria social en el sector cooperativo. 3. Auditoria social como herramienta de control y vigilancia en el marco de un modelo de gobernanza cooperativa. 4. Metodología. 5. Resultados. 6. Conclusiones. 7. Agradecimientos. 8. Referencias bibliográficas.

Cómo citar. Rubio-Rodríguez, G.A.; Santos, F.A. (2021) Auditoría social en las organizaciones del sector cooperativo: precedente de notable escenario de gobernabilidad. REVESCO. Revista de Estudios Cooperativos, vol. $138, \quad$ e73868. https://dx.doi.org/10.5209/reve.73868. 


\section{Introducción}

Las empresas del sector cooperativo al evaluar el cumplimiento de los principios sociales, utilizan como herramienta la auditoría social, la cual persigue valorar, medir y controlar la gestión de los objetivos en beneficio de sus asociados y de la comunidad (Barba-Bayas y Gavilánez-Vega, 2016); es decir, evaluar la eficiencia social, el cumplimiento de los objetivos sociales y el comportamiento ético, de manera que la empresa cooperativa logre optimizar los corolarios en materia social y solidaria y, asimismo, rendir cuenta de éstos a los agentes de interés involucrados con el objeto social de la organización.

Por su parte, las cooperativas de ahorro y crédito consiguen apoyarse en el decreto reglamentario 302 (2015), para facilitar la aplicabilidad de la auditoría social en Colombia. Este decreto presenta el marco técnico de las normas de aseguramiento de la información, que contiene entre otras, las normas internacionales de auditoría. Así las cosas, cada organización cooperativa en cabeza de la dirección general, tendrá la responsabilidad de salvaguardar y de velar por evitar incurrir en actos o en omisiones que afecten la estabilidad económica o el prestigio social de la misma.

El cumplimiento del decreto antes señalado, corresponderá al auditor interno, las juntas de vigilancia y demás comités que guarden competencia para tal efecto. Al mismo tiempo, estos entes de control velarán por el uso de los rubros de cada uno de los fondos de destino específico en las cooperativas de ahorro y crédito, como también del impacto que genere el balance social derivado de éstas. Por consiguiente, cada balance social debe contener información que posibilite gestionar socioeconómicamente la entidad cooperativa, al grado de convertirse en una herramienta que facilita la medición y rendición de cuentas en este aspecto.

Del mismo modo, el papel que desempeña la auditora social frente a la gobernabilidad en el contexto cooperativo, tiene su asiento en la forma como éstas organizaciones orientan su funcionamiento y en la forma como están asumiendo roles y responsabilidades. Por tanto, en la medida que se precisen correctas prácticas de gobernabilidad, se evidenciarán memorables estipendios en beneficio de quienes las ejerciten (Becchetti, Ciciretti y Hasan, 2015). Bajo esta premisa, las actividades que conduzcan al correcto desempeño de la organización, gozarán de conspicuos controles a la gestión en cumplimiento de los objetivos.

En cuanto al contenido del manuscrito, éste pretende reflejar el alcance que arroga la auditoría social al interior de las organizaciones del sector cooperativo, específicamente en las cooperativas de ahorro y crédito; asimismo, revelar la significancia que adopta este tipo de auditoría cuando es utilizada como herramienta para conquistar balances sociales integrales de positivo impacto, con el fin de incrementar la confianza de los usuarios, los colaboradores y, en general de todos aquellos agentes de interés que afectan directa o indirectamente la entidad.

De igual forma, es necesario acotar que las auditorías se desarrollan en un contexto empresarial, cuya función reside en develar el comportamiento organizacional que, para efectos de este trabajo, es de carácter social. Sin embargo, no se desconoce que las auditorías desnudan acertadamente, en muchas ocasiones, las actuaciones de las organizaciones, evidenciando serios problemas de gobernabilidad. De ahí, que el resultado de este ejercicio investigativo también fulgurará la manera en que los órganos máximos de dirección están orientando las cooperativas de las que forman parte.

Siguiendo con el epítome del escrito, se presenta desde una perspectiva teórica, una reflexión acerca de la forma como influye la auditoría social en el sector cooperativo; a su vez, enseña una descripción general de la auditoría social como instrumento indispensable para controlar el uso de los recursos que se encuentran en los fondos de destino específico de este tipo de organizaciones.

Finalmente, se hace un breve análisis respecto el destino y uso de los recursos de cada uno de los fondos de destino específico, tomando como base las cooperativas especializadas de ahorro y crédito que reportan información financiera a la superintendencia de economía solidaria en Colombia.

\section{Acepción e importancia de la auditoría social en el sector cooperativo}

Teniendo en cuenta que concurren diferentes circunstancias en las cuales la corrupción y la irresponsabilidad social son una realidad innegable en la operatividad de las entidades privadas y públicas de disimiles sectores económicos (Saavedra, 2011), la auditoría social se descubre como un instrumento ideado para promover la transparencia, la claridad en los procesos, la confianza en los asociados y el cumplimiento de los principios y valores cooperativos, los cuales se reflejan directamente en la información contable y financiera comprendida en los estados financieros.

La auditoría social para el sector cooperativo, según Aleman, Rivera y Labrador (2010), se define como el mecanismo que consiente a una organización cooperativa justipreciar su comportamiento ético, así como el acatamiento de los objetivos sociales, y dar cuenta de ellos a las personas comprometidas e involucradas con el objeto social de dicha organización. En consecuencia, estas empresas deben demostrar con su acción diaria las razones por las cuales fueron creadas; es decir, bajo la gestión de unos principios, los mismos que orientan su funcionamiento. 
Por lo anterior, es necesario que los modelos de gobernanza cooperativo sean intervenidos a través de la auditoría social, toda vez que, al ejercer el control y la medición a la gestión de los principios cooperativos y objetivos sociales, procederán los resultados en favor de los asociados y de la comunidad en general (Ruiz y Retolaza, 2005). En contraste, al evaluar la gestión social y hallar falta de integralidad, pone de manifiesto el cumplimiento de objetivos y políticas trazadas, por estar encaminadas a resolver problemas principalmente vinculados con actividades económico-productivas (Mancilla y Saavedra, 2015).

La realización de una auditoría social tiene como propósito construir un balance social, (AsencioGallardo, 2015), es decir, reúne, sistematiza y evalúa la información que forja impacto social en la organización; asimismo, aborda impactos positivos y negativos del periodo objeto de análisis, e incluye aspectos relevantes de la responsabilidad social. La responsabilidad social no se limita a la relación trabajadores-asociados, sino, que está dirigida al entorno en el cual opera la empresa. Esta concepción da lugar a un balance social integral (Diaz, Marcuello y Marcuello, 2012).

Añádase a esto que, al igual que un balance económico, el balance social es evaluado con base a unos principios; el primero, es la evaluación del comportamiento ético, que consiste en examinar el control ético de los directivos de la empresa cooperativa (Monserrat, 2015); seguidamente, se analiza el comportamiento de la organización con los stakeholders o grupos de interés, es decir, cuál es la inversión que la organización destina para el desarrollo de su grupo social de interés (trabajadores, asociados, comunidad en general), por medio de la evaluación de la calidad de los servicios prestados y suministro de información útil y confiable (Correa, 2010).

De otra parte, aunque no existe un modelo estándar para adelantar un balance social en Colombia, existe un modelo que se presentó inicialmente en 1987 por la Asociación Nacional de Industrias (ANDI), la Organización Internacional del Trabajo (OIT) y la Cámara Junior de Colombia (CJC) (Correa, 2010), el cual se ilustra en la figura número 1.

Figura. 1. Modelo de balance social.

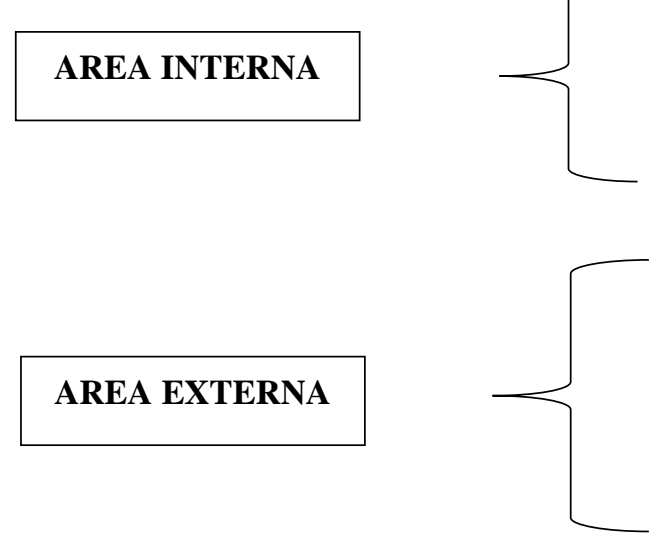

Respuesta y acciones de la empresa hacia sus trabajadores y asociados en tres campos: características socio-laborales, servicios sociales, integración y desarrollo.

Se refiere al cumplimiento de la responsabilidad social con los demás interlocutores sociales que se relaciona en tres aspectos: relaciones con la comunidad, relaciones primarias (familias) y relaciones con otras instituciones.

Fuente: Elaboración propia basado en Caballero y Balaguer (2009)

\section{Auditoría social como herramienta de control y vigilancia en el marco de un modelo de gobernanza cooperativa}

La auditoría social como herramienta, se precisa al realizar su labor de vigilancia y control sobre las labores operativas, administrativas, de toma de decisiones en beneficios comunes conducentes a los asociados (De Lima, Cosenza y Llena, 2015). Respecto la fijación y cumplimiento de las políticas de control y vigilancia establecidas en los estatutos, actas de asambleas y demás documentos, se establece la utilización de recursos financieros para favorecer la labor de responsabilidad social a todos los asociados.

Por su parte, un modelo de gobernanza, según Serna y Rubio-Rodríguez (2016), está ideado por la orientación, el control y la asignación de roles y responsabilidades que son asumidas por determinado tejido empresarial. Ciertamente, para hacer efectiva esta ideación es ineluctable demandar el acompañamiento del recurso humano; toda estructura que acaezca en los niveles administrativos que la conforman, de personal comprometido y que vele constantemente por el cumplimiento de los objetivos estratégicos, gozará de conductas auditables cuyos corolarios se constituirán en aciertos gerenciales.

Divulgado lo anterior, la relación que guarda la auditoría social con la gobernabilidad cooperativa, radica en que los modelos gerenciales esgrimidos en este tipo de empresas, presenta en sus políticas la adopción y 
cumplimiento de ciertos principios y valores, lo cual supone un comportamiento de quienes las integran acorde a lo que propugnan. No obstante, existen varias conductas irresponsables en el campo social que desvirtúan dichas políticas, dejando en evidencia la forma como operan estas instituciones (Zuluaga y Jaramillo, 2011). Por tanto, los modelos de gobernabilidad reflejarán ciertas conductas, que si bien serán auditadas, no todas saldrán favorecidas.

Siguiendo con los modelos de gobernanza y la forma como consiguen ser auditados socialmente, en Colombia, el Decreto 302 de 2015 declara que la responsabilidad en cumplimento de las disposiciones legales y reglamentarias en una organización, es de la dirección, y también estipula el compromiso del auditor de obtener una seguridad razonable sobre un marco normativo aplicable a dichas disposiciones (Molina, Díaz, Vázquez y Casinelli, 2014). Para efectos del presente estudio, las que apliquen a las cooperativas de ahorro y crédito, las cuales en su información financiera deben reflejar el acato de las mismas de forma verídica, verificable, autentica y comprobable.

En consecuencia, el significado de la auditoría frente a la gobernanza de las cooperativas de ahorro y crédito, es mucho más revelador para los asociados, cuyos intereses se fundamentan en el cumplimento del deber ser de los principios cooperativos (Longinos, Arcas, Martínez y Olmedo, 2012), en los que el bien común prima sobre el bien particular. El acatamiento de dichos principios, al igual que los valores, según Castilla y Gallardo (2014), constituyen la plataforma sobre la cual se cimienta una gobernabilidad cooperativa adecuada, sin injerencia autoritaria de ésta sobre el manejo de los recursos.

Por su lado, los asociados, tal y como lo afirma Carreras y Bastida (2015), se erigen como los sujetos de mayor interés en conocer la rendición de cuentas que prorrumpen las entidades cooperativas; el seguimiento por parte de los asociados como agentes fiscalizadores a las buenas prácticas administrativas, gerenciales y financieras, se evidencia en el cumplimiento del objetivo social y su auditoría. Cualquier incertidumbre causada por malos manejos, irregularidades no cubiertas por entidades de control y observancia del direccionamiento de los excedentes, incurrirá en la insatisfacción de la función social y de la propia naturaleza de la cooperativa (Ramírez-Cacho, Martínez-García y Montoya del Corte, 2012).

Finalmente, el logro de una excelente gestión de auditoría social y de implantar verdaderas prácticas de gobernabilidad, tiene doble objetivo: mejorar la calidad de vida de la comunidad sobre la cual realiza sus trabajos sociales, y la intención de mejorar la calidad de vida de los asociados y los empleados de las cooperativas (San-José y Retolaza, 2012). No obstante, la gobernabilidad perenemente será susceptible de ser auditada, en tanto la información financiera y los informes de gestión presentados por los directivos, facilitarán el fiel reflejo del comportamiento de la entidad cooperativa (Cano, 2016).

\section{Metodología}

Guardada la originalidad de este trabajo, es ineludible indicar que el método de investigación empleado corresponde al analítico, en atención a que se disgregaron solícitamente las partes que componen el todo para analizarlas en detalle (Hernández, Fernández y Baptista, 2014). Una vez separadas cada una de las partes, se procedió a recabar información hasta saturar las variables. En esencia, es una tarea dispendiosa, toda vez que en este ejercicio investigativo concurrieron disímiles órganos administrativos de las entidades cooperativas estudiadas, los cuales fueron entrevistados hasta alcanzar el cumplimiento de los objetivos específicos. A continuación, se precisa los pasos empleados para ello:

Inicialmente, es necesario precisar que en Colombia operan, a corte de diciembre de 2019, 180 cooperativas especializadas de ahorro y crédito (Supersolidaria, 2020), de las cuales se tomaron 7 para el estudio. El criterio de elección corresponde a un muestreo no probabilístico en virtud de la conveniencia, pues, dada la naturaleza de este tipo organizaciones en Colombia, no existen razones sustanciales que permitan la improbabilidad de ser elegidas.

El primer paso consistió en visitar las 7 cooperativas especializadas de ahorro y crédito escogidas como unidades de análisis, con el fin de analizar el reparto de excedentes, el destino y uso de los recursos de los fondos de destino específico, y el cumplimiento de los objetivos sociales. La elección de estas cooperativas se adelantó bajo muestreo por conveniencia, dada la proximidad y la accesibilidad a sus órganos máximos de dirección.

Luego de la elección de las unidades de análisis, se entrevistó a integrantes del consejo de administración, como también al gerente general, de cada una de las mismas. Con la inmersión inicial en estas organizaciones cooperativas, asimismo, se entrevistaron miembros pertenecientes a los diferentes comités de vigilancia o auditoría.

Seguidamente, se prosiguió a analizar ordenadamente toda la pesquisa derivada de las poblaciones muestrales; una forma de ordenar la información reside en elegir las unidades temáticas que tienen en común toda la investigación, para que de esta manera se consiga clasificar la información.

Finalmente, después de llevar a cabo esta clasificación, se establece la matriz de síntesis; ésta matriz se constituye en la herramienta imprescindible para conocer los hechos fragmentados de la 
realidad social; es decir, aquellos asuntos comportamentales de las entidades cooperativas, en cuanto al uso de los recursos de cada fondo de destino específico.

\section{Resultados}

De conformidad al objetivo de este trabajo, el cual residió en resaltar la preponderancia que subroga la auditoría social en las cooperativas de ahorro y crédito, desde la forma como son orientadas y controladas, se encontró que este tipo de organizaciones son las más representativas dentro del grupo de empresas del sector solidario en Colombia (Castaño-Ríos, Zamarra-Londoño y Correa-García, 2014), razón por la cual se tomaron dichas cooperativas, con el fin de reconocer desde la auditoría, la distribución de excedentes, la asignación y uso de los recursos de cada uno de los fondos de destino específico, y el cumplimiento de los objetivos sociales.

En este orden, la tabla número 1 muestra un cuadro con las cifras reportadas por estas entidades en sus estados financieros e informes de gestión durante el año 2018. La tabla, asimismo presenta el tamaño de empresa, el cual está determinado por su constitución patrimonial; también, enseña los fondos sociales pasivos y los fondos de destino específico. Los primeros, atienden los fondos por concepto de educación y de solidaridad, y los segundos, sufragan toda actividad relacionada con las necesidades que demanda el conglomerado social. Tanto los primeros como los segundos cuidan el bienestar de los agentes de interés, como una verdadera práctica de responsabilidad social.

De lo precedente, es necesario aclarar que tanto los fondos sociales pasivos como los fondos de destino específico conforman los fondos sociales. Estos fondos se crean con el propósito de gestar una apropiada atención a las necesidades de la base social de las cooperativas y mejorar la calidad de vida de los asociados, colaboradores y su núcleo familiar. Cada año, los fondos sociales son apalancados financieramente con recursos aprobados por la Asamblea General de Delegados, derivados de los excedentes obtenidos en cada vigencia fiscal.

Así las cosas, los fondos sociales pasivos están destinados para asistir a los asociados de la cooperativa en materia educativa y solidaria. Se entiende por apoyo educativo todos los programas orientados a la formación de los asociados y los colaboradores, y lo solidario, a la ayuda económica dirigida a los asociados y sus familiares dependientes, en situaciones especiales tales como calamidades domesticas o circunstancias de particular dificultad. En cuanto los fondos de destino específico, hacen referencia a los proyectos cuyo resultado beneficia a una colectividad.

Tabla.1. Información financiera - Cooperativas de ahorro y crédito

\begin{tabular}{cccc}
\hline \multicolumn{4}{c}{ Cifras estimadas en pesos colombianos } \\
\hline Empresa & Tipo empresa & Fondos sociales pasivos & Fondos destino específico \\
\hline A & Grande & 887.571 .207 & 8.525 .408 \\
\hline B & Mediana & 256.538 .661 & 7.352 .898 \\
\hline C & Grande & 827.140 .000 & 4.527 .876 \\
\hline D & Pequeña & 25.233 .889 & - \\
\hline E & Grande & 917.890 .700 & 5.459 .002 \\
\hline F & Grande & 934.870 .123 & 9.341 .293 \\
\hline G & Pequeña & 38.482 .338 & - \\
\hline
\end{tabular}

Fuente: Elaboración propia a partir de Superintendencia de la Economía Solidaria (2020)

Con la información financiera registrada, se pretende conocer el recurso cuyo destino atiende los fondos sociales (pasivos y de destino específico). Para ejemplificar la forma como se distribuyen los fondos sociales, el artículo 54 de la ley 79 (1988) reza que, de los excedentes cooperativos, "un 20\% como mínimo se debe destinar para crear y mantener una reserva de protección de los aportes sociales; un $20 \%$ mínimo para el fondo de educación, y un 10\% mínimo para un fondo de solidaridad"; el 50\% restante se reserva para la revalorización de aportes.

Entonces, dichos excedentes se distribuyen en los porcentajes antes mencionados, los cuales proveen a los fondos sociales pasivos y a los fondos de destino específico. Entre este último tipo de fondos se encuentran los fondos de: recreación, apoyo a proyectos especiales, bienestar social, etcétera. Estos fondos, permiten visualizar la cantidad monetaria conducente para cada actividad, como producto del desarrollo del accionar económico. Por su parte, los excedentes cooperativos permiten, entre otros, revelar la forma como ha sido distribuido el recurso, en este caso, dentro de las diferentes prontitudes de orden social. 
Así la cosas, nótese que las empresas grandes A y $\mathrm{F}$ presentan mayor concentración de recursos en los fondos de destino específico, lo cual es propio por los excedentes que genera. Sin embargo, las empresas $\mathrm{C}$ y E han destinado menos recursos, incluso, la empresa mediana B sorprendentemente ha invertido más en este concepto. Esto obedece a que ha demandado mayor número de actividades en beneficio del personal asociado, implicando un más alto desembolso de dinero.

Se observa, además, que el valor de los fondos de destino específico de la empresa D y G, no registra saldo, debido a que fueron totalmente agotados durante el año en las actividades de los fondos sociales pasivos; este caso, generalmente se refleja en las pequeñas empresas donde la utilización de excedentes se destina concretamente a proveer las actividades que por ley deben apropiar. El problema que se detecta aquí, es que al cubrir lo demandado por la norma legal, se deja desprotegida toda acción que surja de un improvisto, o que conduzca a mejorar el bienestar del asociado.

Según la información tomada de las notas a los estados financieros e informes de gestión de las cooperativas analizadas, se logró evidenciar que el proceso de selección de los fondos proveídos por los excedentes del ejercicio fiscal, se adelantó de acuerdo a los porcentajes establecidos para el reparto de excedentes, determinado por la ley 79 de 1988. En este sentido, el rol asumido por la gerencia cooperativa adopta una conducta comprometida con el buen uso de los recursos, y no necesariamente por el hecho de cumplir con lo estipulado por la ley, sino, por destinar parte de los excedentes a actividades de beneficio social, diferentes a las consideradas en los fondos de educación y solidaridad.

En concreto, las empresas cooperativas estudiadas cuentan con órganos de dirección caracterizados por cumplir con la custodia de las actividades en materia social. Las actuaciones honestas de quienes tienen a su haber la responsabilidad indelegable de orientar este tipo de entidades, suscitan apreciables afectos. Bajo esta conducta, todo proceso sistemático que conduzca a evaluar el accionar de una organización, por parte de un comité de auditoría o quien haga sus veces, se puede señalar como un indicador de eficacia de gestión social. Así que, las organizaciones cooperativas, deben propender por alcanzar comprometidamente su objeto social; esta es su esencia, su naturaleza, su razón de ser.

A continuación, se presenta el proceder de las cooperativas estudiadas, con relación al recurso económico destinado para atender diferentes actividades de carácter social.

Cooperativa A: Los rubros de las cuentas de pasivo de los fondos sociales reflejados en la información reportada por las cooperativas, corresponden al fondo de solidaridad y educación. Para el caso específico de la cooperativa A, el fondo de solidaridad fue utilizado en el pago de auxilios de salud de los asociados, tales como hospitalización y asistencias de laboratorio; de igual forma, se destinó para el pago de ayudas por calamidad doméstica y enfermedad catastrófica.

Cooperativas B y C: Los saldos del fondo de solidaridad y de educación fueron usufructuados en actividades propias de cada uno de los fondos enunciados en los estatutos de la cooperativa; estos son, inversión en educación formal y auxilios en materia de salud y calamidad doméstica de los asociados.

Cooperativa D: En atención al estudio de la información contable y financiera de la empresa D, registra fondos de destino específico que fueron invertidos en la instalación de rejas, mallas, chapas de seguridad en los salones y dotación de mesas y sillas para los niños de pre-escolar de una institución educativa; estas acciones se abordaron desde un proyecto autorizado debidamente por el Ministerio de Educación Nacional de Colombia. La población beneficiada estaba concentrada en sujetos de bajos recursos, pertenecientes en su mayoría a los estratos más exiguos.

Cooperativa E: En algunas organizaciones, como es el caso de la cooperativa E, se advierte falta de información para los usuarios y asociados interesados; no se evidencia la elaboración de un balance social, que permita determinar y conocer cuál es el destino específico de los recursos del fondo, a fin de forjar una auditoría social que funja como base para evaluar el cumplimiento de metas sociales y el beneficio que tributan a los asociados de la cooperativa y la comunidad en general.

Cooperativa F: Los saldos del fondo de educación fueron invertidos en el $100 \%$ en proyectos educativos aprobados por el Ministerio de Educación Nacional; por tanto, las instituciones educativas fueron las beneficiadas con la ejecución de este rubro.

Cooperativa G: El fondo de educación concentró la inversión en 2 instituciones educativas, atendiendo la dotación de pupitres, tableros, armarios, mesas, mejoras locativas, y la construcción de una cubierta metálica para la sala de profesores; ambas instituciones con población escolar de bajos recursos económicos. Las obras realizadas beneficiaron a un importante grupo de niños y niñas, contribuyendo a hacer más grata su 
permanencia en los espacios escolares. Conforme a la ley, este proyecto contó con la autorización previa de la secretaría de educación municipal y del Ministerio de Educación Nacional.

Consideraciones generales: Cabe señalar que, en la cuenta de fondos sociales, algunas cooperativas crearon fondos nuevos, pero de igual forma poseen destino específico de acuerdo al objetivo social de la cooperativa y el bienestar de los asociados; este es el caso de la cooperativa B, la cual creó el fondo social para otras prontitudes, tocantes con la realización de actividades en materia social, cultural y recreativa, conducentes a la comunidad.

También vinculante es mencionar, que los valores destinados para el fondo de solidaridad no son suficientes para satisfacer las necesidades de los asociados que concurren al comité de solidaridad; en estos casos, la entidad cooperativa debe apoyarse en sus recursos propios; tal fue el caso de la cooperativa G, cuyos asociados se beneficiaron de estos recursos para atender: calamidad doméstica, incapacidad laboral, enfermedad grave del asociado, su cónyuge y sus hijos menores, invalidez, nacimiento de hijos, deterioro descomunal de la vivienda y fallecimiento de un asociado, sus padres, hijos o cónyuge.

Por lo expuesto precedentemente, los hallazgos confirman que la auditoría social cuando es utilizada como auténtica herramienta para promover la transparencia en los procesos, suscita confianza en el cumplimiento de los principios y valores cooperativos. Esto, fundamentado en la implementación de las normas de aseguramiento de información, cuyos lineamentos son claros respecto las responsabilidades que deben arrogar los auditores, el gobierno corporativo, y los asociados en general. Su labor, expresamente consiste en acatar íntegramente las disposiciones legales para lograr una seguridad razonable en la información tanto financiera como social.

Expuesto lo anterior, a diferencia de quienes actúan con base a principios y valores, imperan agentes de interés de la organización cooperativa que no ostentan igual comportamiento, por el contrario, su conducta se traduce en la obtención de estipendios, como producto del lavado de activos mediante el ejercicio cooperativo (Revista Portafolio, 2018). Las derivaciones de estas actuaciones, están soportadas en protervas pericias de gobernabilidad. Un gobierno eficiente, entre sus múltiples finalidades, persigue conquistar información financiera genuina y confiable, con la propensión de alinearla en favor de causas comunes.

Por último, los resultados de este trabajo concuerdan en que las cooperativas objeto de estudio, de acuerdo a lo expresado por Rendón-Álvarez, Rodríguez-Bolaños y Riscos-Gómez (2013), están operando, básicamente, sujetas al derecho constitucional de asociarse con fines útiles y a los principios cooperativos; de igual forma, su constitución provee resultados en común que redundan en beneficio de poblaciones desfavorecidas. Por tanto, la capacidad de las cooperativas consiente la posibilidad de transfigurar los sectores en donde llevan a cabo sus prestezas.

Cabe destacar, que no se halló bajo una mirada empírica, estudios en Colombia análogos a este trabajo; no obstante, Mancilla y Saavedra (2015), afirman que para el sector cooperativo, un buen gobierno corporativo representado por el consejo de administración, es aquel que vela por instaurar un comité de auditoría que procese al interior de la organización, información financiera relevante y confiable. Con relación a esta premisa, no es ininteligible puntualizar que las cooperativas de ahorro y crédito estudiadas, evidenciaron un patrón de conducta acorde a la acepción propia de su naturaleza, como producto del mecanismo de control y vigilancia ejercido a través de los órganos competentes.

\section{Conclusiones}

Respecto al análisis perpetuado a las cooperativas especializadas de ahorro y crédito, que reportan información financiera a la superintendencia de economía solidaria en Colombia, se entrevé que todos los recursos de los fondos de destino específico se utilizaron en actividades taxativas de cada uno de los fondos, relacionadas con educación formal, salud, auxilios funerarios y disimiles prontitudes en favor del asociado; asimismo, se evidencia el cumplimiento del porcentaje de distribución mínimo de los excedentes estimados para los fondos sociales establecidos y regulados por la ley 79 de 1988.

También, se observó que las organizaciones cooperativas estudiadas guardan celeridad en el cumplimiento de la norma, especialmente, cuando se trata de evaluar el compromiso de orientar procesos de eficiencia social. Así las cosas, elaboran balances sociales para comunicar a la sociedad en general el impacto y la evolución de resultados. Estos resultados están representados en las inversiones que son destinadas a cada fondo específico, con el propósito de sufragar las diferentes actividades. Esta conducta da cuenta del eficiente manejo de los recursos financieros, y la imponderable dirección del recurso humano, la cual enmarca un ejemplo de modelo de gobernabilidad.

Se colige en general que, dentro del concepto de gobernabilidad, el éxito de la auditoría social que certifica la ejecución adecuada de los recursos con asignación específica, tiene como causa raíz la actuación del comité de auditoría, quien funge como asesor del consejo de administración de la entidad cooperativa, y ejercita sus funciones de control y seguimiento con disciplina y rigurosidad. Este comité fundamenta su 
accionar en un plan de auditoría que se forja con la supervisión y evaluación de los procesos y resultados, asegurando así los logros institucionales, los cuales son reconocidos por los miembros de las organizaciones objeto del presente estudio.

\section{Agradecimientos}

Los autores desean agradecer a la Universidad para la Cooperación Internacional, por el apoyo en la dirección de la tesis doctoral titulada "Factores influyentes en la conducta de los modelos de gobernabilidad en empresas del sector cooperativo", de la cual se derivó este manuscrito.

\section{Referencias bibliográficas}

Asencio-Gallardo, G. (2015) El Balance Social como una herramienta válida para representar la Responsabilidad Social en las Empresas Chilenas. Revista Ciencia UNEMI, Vol. 8, № 16, pp. 87-96.

Aleman, A., Rivera Rodriguez, J. L., y Labrador Machin, C. A. (2010) Responsabilidad y Balance social en las empresas cooperativas. Revista de Ciencias Sociales, Vol. 14, № 1, pp. 9-19.

Barba-Bayas, D. y Gavilánez-Vega, M. (2016) Gestión social en las Cooperativas de Ahorro y Crédito de una provincia ecuatoriana. Revista Ciencia UNEMI, Vol. 9, № 19, pp. 30-38.

Becchetti, L., Ciciretti, R. y Hasan, I. (2015) Corporate social responsability, stakeholder risk, and idiosyncratic volatility. Journal of Corporate Finance, No 35, pp. 297-309.

Caballero Caballero, I., y Balaguer Vegas, A. I. (2009) Instituto Nacional de Seguridad e Higiene en el Trabajo. Obtenido de http://www.insht.es/.

Cano, A. (2016) La adopción de las NIIF/IFRS en Colombia más que un proceso contable un tema de gobernabilidad corporativa. Revista Espacios, Vol. 37, № 24, pp. 8-17.

Carreras, L. y Bastida, R. (2015) Estudio sobre la rendición de cuentas en materia de responsabilidad social: el balance social. CIRIEC-España, Revista de Economía Publica, Social y Cooperativa, No 84, pp. 251-277.

Castaño-Ríos, C., Zamarra-Londoño, J. y Correa-García, J. (2014) Efectos financieros en una cooperativa colombiana por la implementación de estándares internacionales de información financiera para las pyme en su balance de apertura. Cuadernos de Contabilidad, Vol. 15, №38, pp. 427-458.

Castilla, F. y Gallardo, D. (2014) La revelación social en sociedades cooperativas: una visión comparativa de las herramientas más utilizadas en la actualidad. REVESCO. Revista de Estudios Cooperativos, Primer Cuatrimestre, No 114, pp. 86-112. DOI: http://dx.doi.org/10.5209/rev_REVE.2014.v114.44291.

Correa Jaramillo, J. G. (2010) Evolución histórica de los conceptos de responsabilidad social empresarial y balance social. Semestre Económico, $\mathrm{N}^{\circ} 10$, pp. 1-10.

Decreto 302. Diario Oficial No. 49.431 de la Republica de Colombia, Bogotá D.C. (20 de febrero de 2015) Marco técnico normativo para las normas de aseguramiento de la información. Obtenido de http://www.alcaldiabogota.gov.co/sisjur/normas/Norma1.jsp?i=60905.

De Lima, M., Cosenza, J. y Llena, F. (2015) La auditoría social como mecanismo de control de la responsabilidad social de las empresas: la metodología de Theodore J. Kréps. Contabilidad y Negocios, Vol. 10, № 19, pp. 84-99.

Diaz, M., Marcuello, C., y Marcuello, C. (2012) Empresas sociales y evaluación del impacto social. CIRIEC-España, Revista de Economía Pública, Social y Cooperativa, No 75, pp. 178-198.

Hernández, R., Fernández, C. y Baptista, M. (2014) Metodología de la Investigación. México: McGraw Hill. p. 613.

Ley 79. Diario Oficial No. 38.648 de la Republica de Colombia, Bogotá D.C. (10 de enero de 1988) "Por la cual se actualiza la Legislación Cooperativa". http://www.alcaldiabogota.gov.co/sisjur/normas/Norma1.jsp?i=9211.

Longinos, J., Arcas, N., Martínez, I. y Olmedo, I. (2012) Transparencia, gobierno corporativo y participación: claves para la implantación de un código de conducta en empresas de economía social. REVESCO. Revista de Estudios

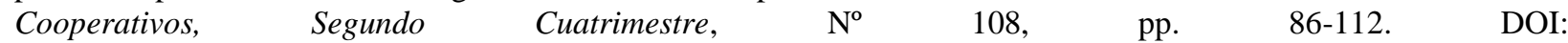
http://dx.doi.org/10.5209/rev_REVE.2012.v18.39588.

Mancilla, M. y Saavedra, M. (2015) El gobierno corporativo y el comité de auditoría en el marco de la responsabilidad social empresarial. Contaduría y Administración, $\mathrm{N}^{\circ} 60$, pp. 486-506.

Molina Llopis, R., Díaz Becerra, O., Vázquez, J., y Casinelli, H. (2014) El proceso de convergencia con las Normas Internacionales de Información Financiera en España, Perú y Argentina. Contabilidad y Negocios, Vol. 9, № 18, pp. $5-26$.

Monserrat Botello, M. (2015) Ética de la empresa: gestión de los valores contra la corrupción en las empresas. Trabajo Final de Grado. Universidad Jaime I, Castellón de la Plana, España.

Ramírez-Cacho, S., Martínez-García, F., y Montoya del Corte, J. (2012) La adopción de las reformas cualitativas sobre materialidad en auditoría. Evidencia empírica de su uso y consecuencias en México y España. INNOVAR, Vol. 22, $\mathrm{N}^{\mathrm{o}} 43$, pp. $165-178$.

Rendón-Álvarez, B., Rodríguez-Bolaños, J. y Riascos-Gómez, P. (2013) Análisis del impacto en el capital institucional de las cooperativas de ahorro y crédito frente a la aplicación de las NIIF. Caso cooperativas del Valle del Cauca 
[número especial: Cuatro años de convergencia contable: resultados, transformaciones y retos]. Cuadernos de Contabilidad, Vol. 14, No 36, pp. 881-901.

Revista Portafolio (14 de junio de 2018) Perú aprueba ley para supervisar cooperativas ante lavado de activos. Obtenido de http://www.portafolio.co/internacional/peru-aprueba-ley-para-supervisar-cooperativas-ante-lavado-deactivos-518108.

Ruiz Roqueñi, M., y Retolaza, J. L. (2005) La auditoría social como instrumento de posicionamiento de la economia solidaria. CIRIEC-España, Revista de Economía Pública, Social y Cooperativa, No 53, pp. 285-304.

Saavedra, M. (2011) La Responsabilidad Social Empresarial y las finanzas. Cuadernos de Administración, Vol. 27, N 46, pp. 39-54.

San-José, L. y Retolaza, J. (2012) Participación de los stakeholders en la gobernanza corporativa: fundamentación ontológica y propuesta metodológica. Universitas Psychologica, Vol. 11, № 2, pp. 619-628.

Serna, H. y Rubio-Rodríguez, G. (2016) La gobernabilidad en el sector cooperativo: una reflexión acerca de su verdadera implementación. Revista Virtual Universidad Católica del Norte, № 48, pp. 239-256.

Superintendencia de la Economía Solidaria (2020) Estados Financieros de las Cooperativas de Ahorro y Crédito. Obtenido de http://www.supersolidaria.gov.co/es/content/cooperativas-de-ahorro-y-credito-2020.

Zuluaga, J. J., y Jaramillo, P. A. (2011) Ética empresarial y códigos de buen gobierno. Tesis doctoral. Escuela de Ingeniería de Antioquia, Medellín, Colombia. 